\title{
Clinical and genetic aspects in the Ehlers Danlos syndrome
}

\author{
Elena Silvia Shelby ${ }^{1}$, Madalina Cristina Leanca ${ }^{1}$, Serban Hamei², \\ Liliana Padure ${ }^{1,3}$, Andrada Mirea ${ }^{1,3}$ \\ 1"Dr. Nicolae Robanescu“ National Clinical Center for Children's Neurorecovery, Bucharest, Romania \\ 2"Marie Skodlowska Curie" Children's Hospital, Bucharest, Romania \\ 3"Carol Davila" University of Medicine and Pharmacy, Bucharest, Romania
}

\begin{abstract}
Introduction. Ehlers Danlos syndrome is a group of hereditary diseases of the connective tissue with a combined prevalence of 1 in 5,000 cases which have in common articular hyperlaxity and skin abnormalities. In some types, rapid diagnosis can mean saving the life of the patient.

Case presentation. We will present the case of a two year old girl which was brought for genetic consult with the suspicion of Ehlers Danlos syndrome. The patient had joint and skin hyperextensibility, velvety skin with a tendency of bruising, congenital hip luxation with a failed surgical intervention, talus varus and severe motor development retardation. Genetic testing revealed the substitution c. $1780 \mathrm{C}>\mathrm{T}, \mathrm{p}$.(Arg594*) in the COL5A1 gene which leads to the apparition of a premature stop codon, the mutation being class 2 (potentially pathogenic).

Conclusions. Up until now, this variant has been reported in the literature in only two patients, ours being the third. All three cases correspond to the classic type Ehlers Danlos syndrome.
\end{abstract}

Keywords: Ehlers Danlos syndrome, joint hyperlaxity, COL5A1

\section{INTRODUCTION}

Ehlers Danlos syndrome is a group of hereditary diseases of the connective tissue (1) with a combined prevalence (for all types) of 1 to 5,000 cases (2). The joints, skin, blood vesses or other organs are affected (2).

According to the classification made by Beighton and his collaborators in 1998 (the Villefranche Nosology) (3), Ehlers Danlos syndrome was divided into six major types which have in common joint hyperlaxity and skin abnormalities, frequent dilatation of the aorta and rarely, rupture of the aorta (for example, in the vascular type) (1). In 2017, Malfait and his colaborators (4) revised the classification criteria according to the molecular basis responsible of the etiology, dividing the Ehlers Danlos syndrome into 14 subtypes (5), among which the molecular mechanism is currently known for 13 (6).

The common characteristics of all types of Ehlers Danlos syndrome are joint hyperlaxity and skin ab- normalities (1). The hypermobility is evaluated, for all types, using the Beighton scale, where according to the degree of mobility of some joints, a diagnostic score is established; if it's above or equal to $5 / 9$, the patient is considered to have hypermobility $(1,7)$.

According to the International Diagnostic Criteria established in 2017, Ehlers Danlos syndrome is divided into the following subtypes: classic, vascular, with arthrochalasia, with dermatosparaxis, cardiacvalvular (produced by abnormalities of the primary structure and processing of the collagen), kyphoscoliotic (produced by abnormal packing and polymerisation of the collagen), classic-like and myopathic (produced by dysfunctionalities of the structure and function of the myomatrix - the basal membrane of the skeletic muscle) $(5,8,9)$, spondylodysplastic and musculocontractural (produced by dysfunctions of the glycosaminoglycan biosynthesis), periodontal (produced by dysfunctions of the complement pathway), spondylodysplastic SLC39A13 and brittle cor- 
nea syndrome (produced by dysfunctions of the intracellular processing), as well as hypermobile (in which the mechanism of the disease is not known yet) $(5,8)$.

Collagen is a fibrillary protein present in the structure of the human connective tissue, especially in the joints, skin and bones $(10,11)$, being the most abundent protein of the extracellular matrix $(10,12)$ and at the same time the most abundent protein in the body, where it accomplishes an essential role in maintaining the structure and stability of various organs. (13) Up to now, there are 28 types of collagen known $(13,14)$, the most common being type I, which represents over $90 \%$ of the total collagen that enters the composition of the human body (14). The main components of the collagen are glycine, proline and hydroxyproline, forming 3 chains packed as a triple helix $(14,15)$.

Type V collagen is synthetized in various types of mesenchymal cells under the influence of TGF- $\beta$ and other growth factors, and degraded by metalloproteinases and gelatinases, enzymes with a role in extracellular matrix remodelling (16). This type of collagen is found in the tissues which contain type I collagen, where the regulation of the packing process of the heterotypical fibers composed of type I and type V collagen takes place (17). Thus, type V collagen is a component of the corneal stroma, of the bony matrix and of the stroma of the liver, lungs, muscles and placenta (18). The absence of type V collagen was associated with the classic type of Ehlers Danlos syndrome, as well as with the loss of the corneal transparency, while the supraexpression of this molecule is associated with pathological processes such as fibrosis, cancer, inflammation or atherosclerosis (16).

The classic type of Ehlers Danlos syndrome is mainly caused by autosomal dominant mutations in the COL5A1 and COL5A2 genes, and, in rarer cases, COL1A1 $(19,20)$, and has as main diagnostic criteria generalized joined hypermobility and skin hyperextensibility with atrophic scars (19), among the minor criteria being velvety skin, tissue fragility and extensibility which can lead to complications such as anal prolapse, hiatal hernia, cervical incompetency, the presence of subcutaneous spheroids (partially calcified formations which appear on the bony prominences), molluscoid pseudotumours (scars which appear in the areas which are frequently subject to pressure, such as elbows or knees), predisposition to bruises, muscle hypotonia, delay in obtaining the motor acquisitions as well as complications of the joint hyperextensibility such as shoulder, hip, patella, clavicle or radius dislocations $(20,21)$. Globally, the classic type of Ehlers Danlos syndrome has a prevalence of 1 in 20,000 (22).

\section{CASE PRESENTATION}

A two year old girl was brought for genetic consult with the suspicion of Ehlers Danlos syndrome. The family and birth history were not significant.

Regarding the medical history, the patient was born with congenital unilateral hip luxation for which surgery was performed at the age of one year and four months. Still, a second surgery was needed, when the patient was one year and seven months old.

The patient had delayed motor milestone acquisitions - she could hold her head at 4 months and a half, could sit independently at 9 months, and had never acquired walking, but she could maintain orthostatism. Regarding the cognitive development, the patient was in the limits of normal.

The mom claimed that the patient does not bruise easily and that she never had hernia, epistaxis or pneumothorax.

At the time of the consult, the patient had never had a heart ultrasound, and the levels of the serum creatine kinases had never been measured.

At the clinical exam, the growth parameters were normal. The patient had a slight facial dysmorphism with hypertelorism and micrognathia. Also, she had a postoperative scar on her right hip. She had joint hyperextensibility (with 6 out of 9 points on the Beighton scale), a velvety, doughy and hyperextensive skin, a slight lower limb asymmetry and bilateral talus varus.

To elucidate the cause, a blood sample $(2 \mathrm{ml}$ on EDTA) was harvested for next generation sequencing - targeted panel responsible for Ehlers Danlos syndrome. The substitution c. $1780 \mathrm{C}>\mathrm{T}$, p.(Arg594*), in the COL5A1 gene, was found in heterozygous state. This substitution is considered a class 2 mutation (likely pathogenic) and leads to the apparition of a premature stop codon. Mutations in this gene were associated with the classic type of Ehlers Danlos syndrome with autosomal dominant inheritance. Up until now, this variant has only been reported in the literature in two other patients, ours being the third. All three cases correspond to the classic type Ehlers Danlos syndrome.

\section{DISCUSSIONS}

Currently, in the ClinVar database there are over 100 pathogenic or potential pathogenic known mutations in the COL5A1 gene, among which 16 are missense (23).

The variant identified by us, c.1780 C>T, p.(Arg594*) in the COL5A1 gene, leads to the apparition of a premature stop codon and to the loss of 
function of the protein through the production of a truncated protein, only 593 out of 1,837 aminoacids being codified. So far, the variant was reported in only two other patients (24), being also present in the ClinVar database (25). The variant is absent from the Genome Aggregation Database (gnomAD) (26).

Thus, the only other study in the literature which has reported this variant was made by Malfait and her collaborators in 2005 (24) on 48 unrelated patients which had been clinically diagnosed, based on the criteria defined by the Villefranche Nosology in 1997, with the classic type of Ehlers Danlos syndrome, the inclusion criteria being joint hyperextensibility, the presence of extended atrophic scars and of generalized joint hypermobility. Malfait and her collaborators analysed the presence of type I and V collagen defects in fibroblast cultures from these patients using various methods, such as the fluorographic examination of radioisotope-marked type $\mathrm{V}$ collagen, to identify null, nonfunctional alleles of type $\mathrm{V}$ collagen, the electrophoresis of the COL1A1, COL1A2, COL5A1 and COL5A2 genes using the SSCP (single-strand conformation polymorphism) and CSGE (conformation sensitive gel electrophoresis) methods to identify the migration abnormalities and, thus, the mutations in these genes, linkage studies involving mutations in the COL5A1 genes using intragenic and extragenic microsatellites utilizing DNA or complementary DNA obtained through the reverse-transcription of RNA, in order to analyse the segregation pattern of the variants in the patients' families, the amplification and sequencing of DNA to identify certain mutations in type $\mathrm{V}$ and type I collagen, as well as the analysis of three polymorphic sites in the complementary DNA of COL5A1 gene to identify the polymorphisms at this level.

Out of the 48 patients, in 18 (representing 37.5\%) a nonfunctional allele of collagen $\mathrm{V}$ was identified. In spite of the identification of a premature stop codon in all of the patients, the expression of the null COL5A1 allele was not totally absent.

The mutation identified by us, c. $1780 \mathrm{C}>\mathrm{T}$, p. $(\operatorname{Arg} 594 *)$ in the COL5A1 gene, is present in the EDS 15 and EDS 20 patients of Malfait's study (p.R594X). The mutation affects codon 16 of the COL5A1 gene, the substitution leading to the apparition of a premature stop codon. Interestingly, the initial studies using the SSCP and CSGE electrophoresis of COL5A1 using did not identify migration abnormalities in patient EDS15, the mutations present in this patient being subsequently identified using dHPLC (denaturing high performance chromatograpy). Similarly to the patient identified by us, the other two patients with the c.1780C $>$ T, p.(Arg594*) mutation in the COL5A1 gene, EDS 15 and EDS 20, respectively, from Malfait's study, are females. Patient EDS15 was, at the moment of the study, 11 years old, and patient EDS20, 19 years old. Patient EDS15 had a spontaneous mutation, while at patient EDS20 the mutation was inherited (the inheritance mode being autosomal dominant). Because the parents of our patient do not want yet to be tested, we cannot know exactly how the variant segregates. Still, based on the anamnesis, we can assume that the mutation is de novo, the family history being unsignificant.

The case presented by us has in common with the EDS15 and EDS20 patients the joint hyperextensibility and the presence of the soft, velvety skin (the patient being, at the moment of the study, two years old) which was relatively hyperextensible (it could be extended about $1,5 \mathrm{~cm}$ at the level of the distal portion of the forearms and the dorsal portion of the hands), as well as of the atrophic scars (the patient had a postsurgery scar on the right hip). Unlike patients EDS15 and EDS20 described by Malfait, who presented the tendency to bruising, according to the anamnesis and clinical exam the patient examined by us did not present this predisposition. It must be taken into account, though, the fact that when this study was done she had not acquired walking abilities.

In Malfait's study, patient EDS15 also presents anal prolapse, a characteristic unmet in our patient or in patient EDS20. Besides these attributes, patients EDS15 and EDS20 did not have other relevant clinical features (such as, for example, short stature, blue sclerae, recurrent dislocations or hip or shoulder luxations). Our patient had a slight facial dysmorphism with hypertelorism and micrognathia, congenital unilateral hip luxation and bilateral talus varus.

\section{CONCLUSIONS}

Phenotypically, the patient presents features of the classic type of Ehlers Danlos syndrome, the genetic testing having confirmed the presence of this syndrome.

The present study is important for the pediatric practice as the identified variant is very rare, currently having been reported in the literature in only two patients, the case presented by us being the third. All three patients are females.

Because data presented up to now in the only study which describes patients with this mutation is very scarce and because our patient presents additional phenotypic traits to the ones presented so far (slight facial dysmorphism with hypertelorism and micrognatia, congenital unilateral hip luxation and bilateral talus varus), we believe that the detailed 
presentation of this case can be useful in expanding the knowledge about the classic type of Ehlers Danlos syndrome and about this variant.

\section{REFERENCES}

1. Tinkle BT, Atzinger CL. Ehlers-Danlos Syndrome. In: Cassdy S, Allanson J. Management of Genetic Syndromes. New Jersey: Wiley Blackwell. Third Edition. 2010: 337-361.

2. Genetics Home Reference. [Online]. [Updated 2020]. Available from: URL: https://ghr.nlm.nih.gov/condition/ehlers-danlossyndrome\#statistics.

3. Beighton P, De Paepe A, Steinmann B, Tsipouras P, Wenstrup RJ. Ehlers-Danlos syndromes: revised nosology, Villefranche, 1997. Ehlers-Danlos National Foundation (USA) and Ehlers-Danlos Support Group (UK). Am J Med Genet. 1998;77(1):31-7.

4. Malfait F, Francomano C, Byers P, Belmont J, Berglund B, Black J, Bloom L, Bowen JM, Brady AF, Burrows NP, Castori M, Cohen H, Colombi M, Demirdas S, De Backer J, De Paepe A, Fournel-Gigleux S, Frank M, Ghali N, Giunta C, Grahame R, Hakim A, Jeunemaitre X, Johnson D, Juul-Kristensen B, Kapferer-Seebacher I, Kazkaz H, Kosho T, Lavallee ME, Levy H, Mendoza-Londono R, Pepin M, Pope FM, Reinstein E, Robert L, Rohrbach M, Sanders L, Sobey GJ, Van Damme T, Vandersteen A, van Mourik C, Voermans N, Wheeldon N, Zschocke J, Tinkle B. The 2017 international classification of the Ehlers-Danlos syndromes. Am J Med Genet C Semin Med Genet. 2017;175(1):8-26.

5. The Ehlers-Danlos Society. EDS Types. [Online]. [2020]. Available from: URL: https://www.ehlers-danlos.com/eds-types/.

6. Malfait F, Castori M, Francomano CA, Giunta C, Kosho T, Byers PH. The Ehlers-Danlos syndromes. Nat Rev Dis Primers. 2020;6:63.

7. Benign Joint Hypermobility Syndrome - not just a flexible child. Children's Podiatry Melbourne. [Online]. [2016]. Available from: URL: http://childrenspodiatry.melbourne/index.php/blog/benign-jointhypermobility-syndrome-not-just-flexible-child.

8. Meester JAN, Verstraeten A, Schepers D, Alaerts M, Van Baer L, Loeys BL. Differences in manifestations of Marfan syndrome, Ehlers-Danlos syndrome, and Loeys-Dietz syndrome. Annals of Cardiothoracic Surgery. 2017;6(6):582-594.

9. Ruegg MA, Meinen S, Maier G, Lin S, Yurcencho PG. The role of laminins in myomatrix assembly and skeletal muscle stability. Neuromuscular Disorders. 2013;23(9-10):738-739.

10. Chiarelli N, Ritelli M, Zoppi N, Colombi M. Cellular and Molecular Mechanisms in the Pathogenesis of Classical, Vascular, and Hypermobile Ehlers-Danlos Syndromes. Genes (Basel). 2019; 10(8):609.

11. Avila Rodriguez MI, Rodriguez Barroso LG, Sanchez ML. Collagen: A review on its sources and potential cosmetic applications. Journal of Cosmetic Dermatology. 2017;17.

12. Sorushanova A., Delgado LM, Wu Z, Shologu N, Kshirsagar A, Raghunath R, Mullen AM, Bayon Y, Pandit A, Raghunath M, Zeugolis DI. The Collagen Superfamily: From Biosynthesis to Advanced Biomaterial Development. Adv Mater. 2019;31(1).

\section{Acknowledgement}

The authors would like to thank the carers of the patient for the good collaboration.

\section{Conflict of interest: none declared Financial support: none declared}

13. Kwatra B. Collagen Supplementation: Therapy for Skin Disorders: A Review. World Journal of Pharmaceutical Research. 2020; 9(5):2504-2518.

14. Wu M, Cronin K, Crane JS. Biochemistry, Collagen Synthesis. [Updated 2020 Sep 11]. In: StatPearls [Internet]. Treasure Island (FL): StatPearls Publishing; 2020 Jan-. Available from: https://www. ncbi.nlm.nih.gov/books/NBK507709/.

15. León-López A, Morales-Peñaloza A, Martínez-Juárez VM, VargasTorres A, Zeugolis DI, Aguirre-Álvarez G. Hydrolyzed CollagenSources and Applications. Molecules. 2019;24(22):4031.

16. Mak KM, Png CY, Lee DJ. Type V Collagen in Health, Disease, and Fibrosis. Anat Rec (Hoboken). 2016;299(5):613-29.

17. COL5A1 - collagen type V alpha 1 chain. NCBI Gene. [Online]. [Updated 2020]. Available from: URL: https://www.ncbi.nlm.nih.gov/ gene?Db=gene\&Cmd=DetailsSearch\&Term=1289.

18. DJ Leeming, MA Karsdal. Type V Collagen. In: Karsdal MA. Biochemistry of Collagens, Laminin and Elastin. Second Edition. Elsevier Inc. 2019:51-57.

19. Ritelli M, Venturini M, Cinquina V, Chiarelli N, Colombi M. Multisystemic manifestations in a cohort of 75 classical EhlersDanlos syndrome patients: natural history and nosological perspectives. Orphanet J Rare Dis. 2020;15(1):197.

20. Malfait F, Wenstrup R, De Paepe A. Classic Ehlers-Danlos Syndrome. 2007 [Updated 2018]. In: Adam MP, Ardinger HH, Pagon RA, et al., editors. GeneReviews ${ }^{\circledR}$ [Internet]. Seattle (WA): University of Washington, Seattle; 1993-2020. Available from: https://www.ncbi. nIm.nih.gov/books/NBK1244/.

21. Malfait F, Wenstrup RJ, De Paepe A. Clinical and genetic aspects of Ehlers-Danlos syndrome, classic type. Genet Med. 2010 Oct; 12(10):597-605.

22. Classical Ehlers-Danlos syndrome. Orphanet. [Online]. [Updated 2020]. Available from: URL: https://www.orpha.net/consor/cgi-bin/ OC_Exp.php?lng=en\&Expert $=287$.

23. COL5A1. ClinVar. [Online]. [Updated 2020]. Available from: URL: https://www.ncbi.nlm.nih.gov/clinvar/?term=COL5A1\%5Bgene\%5D.

24. Malfait F, Coucke P, Symoens S, Loeys B, Nuytinck L, De Paepe A. The molecular basis of classic Ehlers-Danlos syndrome: a comprehensive study of biochemical and molecular findings in 48 unrelated patients. Hum Mutat. 2005 Jan;25(1):28-37.

25. NM_001278074.1 (COL5A1): c.1780C>T (p.Arg594Ter). [Online]. [Updated 2020]. Available from: URL: https://www.ncbi.nlm.nih.gov/ clinvar/variation/519624/.

26. COL5A1. gnomAD browser. [Online]. [Updated 2020]. Available from: URL: https://gnomad.broadinstitute.org/gene/ ENSG00000130635?dataset=gnomad_r2_1. 